\title{
Statement of the Problem of Pedagogical Diagnostics in Literature on Pedagogy and School Practice
}

\author{
Tovuz Bakhtiyar Eminli \\ Zagatala Branch of the Azerbaijan Institute of Teachers \\ Zagatala, the Republic of Azerbaijan \\ E-mail: teminli@box.az
}

Received: May 29, $2011 \quad$ Accepted: July 1, $2011 \quad$ doi:10.5539/ass.v7n8p260

\begin{abstract}
The paper is devoted to the investigation of the questions connected with the statement of the problem of pedagogical diagnostics in literature on pedagogy and school practice. The results of the analyses on pedagogical diagnostics in literature on pedagogy and school practice enable us to come to the conclusion that the pedagogical diagnostics, diagnostics of pupils', teachers', school and form masters'work, in general, diagnostics of the work of all school is imperative. It creates favourable opportunities and conditions for a rational construction of a pedagogical process at an educational centre.
\end{abstract}

Keywords: Diagnosis, Pedagogical diagnostics, Psychological diagnostics, Diagnostics of pupils' activity, Diagnostics of teachers' activity

\section{Introduction}

In spite of the term of the pedagogical diagnostics is used in science for the last decade, it has an ancient history. Pedagogical diagnostics serves to increase the quality of a pedagogical process and improvement of activity in every educational institution irrespective of kind of school and it has always been a constituent part of a purposeful, regular and systematic activity. By means of pedagogical diagnostics it is rationally possible to manage with the teaching and training process and to acquire a higher achievement. Teaching of pupils and out-of-school activity is a main object of pedagogical and psychological diagnostics. Pedagogical and psychological diagnostics is carried out with pupils, group of pupils, and staff of pupils as well.

Not only pupils, but also form-masters, teachers, public organizations of a school, leadership of a school, its administration and others may be involved in the pedagogical diagnostics. Moreover in different researches, books, brochures and articles it is paid attention to the diagnostics of development, the diagnostics of pupil's training level, the diagnostics of the ability of learning, the diagnostics of the teaching and good- breeding level and other problems.

\section{Statement of the problem of pedagogical diagnostics in literature on pedagogy and school practice}

Diagnosis is a word of Greek originated. "Dia” - means transparent, "gnosiz” - means knowledge. Diagnostics means a generally used method for obtaining of early information about reviewed object or process (Abbasov, 2008).

Besides A. N. Abbasov such Azerbaijan pedagogues as A. A. Aghayev (Aghayev, 2006), A. Kh. Pashayev, F. A. Rustamov (Pashayev, Rustamov, 2007), L. N. Gasimova (Gasimova, Mahmudova, 2002) and others expressed their opinions in their books on Pedagogy. Moreover A. O. Mehrabov, A. M. Abbasov, Z. Z. Zeynalov, R. Hasanov (Mehrabov et al, 2006), H. H. Ahmadov (Ahmadov, 2006) also stated their opinions about pedagogical diagnostics in their books. Such psychologists as A. A. Alizada, M. A. Hamzayev and others (Alizada, A., 2004; Alizada, A., Alizada, H., 2010; Hamzayev, 1995) devoted some of their researches to the problem of psychological diagnostics as well.

Diagnosis (enquiry) and purposeful, systematic, sequential operation are carried out in the direction of eligibility and troubleshooting in schools of the Republic of Azerbaijan. On the basis of pedagogical diagnostics it is worth researching of personality of a pupil. Formation of integrative property of an individual, practical readiness for a public activity, education, learning, self-education and self-discipline - is accepted as the signs of good-breeding. 
The object of pedagogic diagnostics is arranged with the consistency of integrative property of an individual with the process of educating or breeding and relationship of a pupil to the educational influences.

Propensity to negative properties to be difficult-breeding, breach of the law and juvenile crime are the indicators of low level in the system of family and public education. The most characteristics manifestation of a man is his social essence. It is reflected in the system of his relationship to the world, environment and himself. Existing social processes and appearance of it in each man find its expression in consciousness, is arranged, processed again, as well as the development of natural forces and possibilities of a man are provided. They provide still more development of all obtained in the result of a personal life and education. The issues which is typical of each man (sex, age, type of a nervous system, temperament and etc.) is related with the appearance of social reality (time, social system, relationship of ideology and morality, science and technique, culture and production, education and family).

Notably the object of the pedagogical diagnostics is not only educated, enclosed with education of a pupil (student), but also educating possibilities of the nearest social sphere for it that is forming, developing and educating character of education process.

Just from the first day of training it is important to carry out purposeful, systematic, organizing, continuous, regular work of teaching and educating character with pupils. For achievement of rational result, pupils must comprehensively learn everything.

Pupils have common and personal development parameters. When a pupil comes to school he already has a readably pronounced individuality. That is all complexity and inconsistency of his moral world, his action from a simple to compound, from a little to adult, from a biological to sociological and etc. must be taken into consideration.

What just must be learned in pupils? For the estimation of the knowledge level and manners of pupil it is necessary to be based on certain criteria. It is truth that educating and developing is not identical. Different components of education help to develop personality of a pupil. But we must not forget that people are not alike from point of view of mental, physical, moral development.

In pedagogy, psychology and philosophy the problems about harmonic and detailed development of a person are considered and the scientific-pedagogical and psychological bases for formation of a perfect man are clarified.

The goals of education are specified with interests and properties of the society and individual from the social aspect. The expediency of a person is the main problem of education. If the object of a society answers the objects of a person, the purpose to educate is achieved then.

A number of researches on pedagogical and psychological diagnostics was carried out in foreign countries. In this respect the German pedagogue Karlheinz Ingenkamp's researches attract a special attention.

He drew up the textbook "Pedagogical diagnostics" (Ingenkamp, 1991) where the problems of pedagogical diagnostics were reflected. It should be noted that the author based on the experience of the German schools. The textbook was divided into such a main sections as "The subject of the pedagogical diagnostics and its historical development", "Methodical problems of the pedagogical diagnostics", "Diagnostics of the academic performance", "Diagnostics of the development of intellectual facilities and availability of educating in the system of education", "Diagnostics of the social and economic characters". Unlike other authors K. Ingenkamp adheres to the opinion that "essentially the pedagogical diagnostics numbers as much as all pedagogical activity. Who regularly taught and always tried to identify the results of his efforts ... inherent educator understands difficulties occurred in a teaching process better than others and he responded to them more flexibly (Ingenkamp, 1991).

K. Ingenkamp also expressed his opinion about the methodic criteria of measure properties in social sciences (Ingenkamp, 1991). According to its aspects the measuring results should comply with the requirements of exactness and reliability. The author attaches importance to identifying of measuring results in the pedagogical diagnostics. This is a correct estimation. With this purpose he considers necessary to develop corresponding criteria which make condition for estimation of measuring characteristics and assign among them 1) objectivity; 2) reliability and 3) unity.

It is possible to state the articles by E. A. Mikhaylichev on the topics of "Methodology of diagnostics of breeding" (Mikhaylichev, 2003a) and "Methodology of pedagogical psycho-diagnostics" (Mikhaylichev, 2003b). In the article "Methodology of diagnostics of breeding" by E. A. Mikhaylichev the theoretical bases of diagnosing of breeding are explained primarily (Mikhaylichev, 2003a). According to the author's opinion "The theoretical basis of diagnosing of breeding currently serves typology of educational direction, the constituent 
parts of educational system, essential features of criterial nature, advanced achievements of education in common or in its separate aspects" (Mikhaylichev, 2003a).

E. A. Mikhaylichev's following opinion is of significance that "the considerable complexities of methodological character in the educational diagnostics is also connected with absence of the common interpretation of the term "breeding", by which some of authors mean previous educational activities carried out (Mikhaylichev, 2003a). In the article more attention is paid to the method of definition of acquaintance with dictionary covering of the notion and level of cognition of political terminology and study of social world view by pupils.

The second article ("Methodology of pedagogical psycho-diagnostics") by E. A. Mikhaylichev (Mikhaylichev, $2003 \mathrm{~b}$ ) is devoted to the methods of pedagogical psycho-diagnostics. The author of the article addresses to such opinion of specialists on psycho-diagnostics that teachers may use it without demanding any special knowledge during interpretation, separately, theoretically and psychometrically with basic methods. He includes here the followings: "-preliminarily consult psychologists during selection of a method and when there are certified methods to be used;

- if the method require special professional-psychological knowledge, it is necessary to choose other method or have a corresponding training or involve a professional psychologist or reject the psycho-diagnostics;

- the user complies with all ethic norms in carrying out of research regarding to the respondent and any third parties and as a psychologist is not entitled to betray confidence and must forewarn the respondent about how should be the information used;

- the methods which has not been provided with the standard single-valued instruction, necessary reliability indexes and validity required a parallel use of high-professional expert methods that could not be used by the specialists-non-psychologists and etc.” (Mikhaylichev, 2003b).

Practical psychologists work at schools. At a number of state and non-state higher educational schools of the Republic of Azerbaijan personnel on the speciality of "school psychologist" is prepared. But in accordance with the observations and inquiries studying from the school documents it is obvious that although the activity of more school psychologist is satisfactory, there are some drawbacks in the works of more practical psychologists part of which is related just with the school psychologists: they are lack of professionalism; they demonstrate a theoretical and practical incompetence. Other part of deficiency arises due to interference of a school leadership in the activity of practical psychologists or their indifferent attitude towards this, non-involving them into the work on purpose, non-conditioning (a separate room, necessary equipments and etc.). However this naturally will restrict the activity of school psychologists and bring to negligent and irresponsible attitude towards their duties.

Just in such cases it is obviously that psychological services sometimes become monopolies of a practical psychologist. Although his role first of all consists of management of the given issue. He should manage methodological and methodic provision and management of the given work. The sphere of his activity should be directed to adoption of difficultly detached methods. Certainly the responsibilities of a school psychologist are broad and encompassing. Our aim is not to concentrate our attention on it.

The diagnostics of the activity of pupils, teachers and totally the school should become not only a work of the practical psychologist, but also simultaneously form and school masters, individual teachers. It is no coincidence that E. A. Mikhaylichev in his article "Methodology of pedagogical psycho-diagnostics" (Mikhaylichev, 2003b) offers two main method groups for rational implementation pedagogical psycho-diagnostics. Let us draw attention: "intended for a school psychologist-professional; 2) intended for mass teacher and available for him without any special professional-psychological preparation".

B. Zel'tserman and N. Rogaleva (Zel'tserman, Rogaleva, 2002) paid attention to the pedagogic diagnostics in their researches as well. The authors determined that "in the process of working teachers is confronted with the problem of impossibility to estimate the behavior of all pupils of a class, because the teacher can "observe" the behavior of 4-6 pupils during lesson. Other children of the class stand aside of his observation. This makes the procedure of the expert evaluation extremely long and labor-intensive: it is necessary to design a few diagnostic lessons in order to trace behavior of a certain child group".

B. Zel'tserman and N.Rogaleva (Zel'tserman, Rogaleva, 2002) see the way out in addressing to the method of video-expertise. In the article the nature of the indicated method is clarified. It consists of shooting the behavior of all (or majority) pupils. During shooting the following persons should take part:

1) the cameraman carrying out (organizing) the shooting; 
2) the teacher holding the diagnostic lesson;

3) the expert observing the lesson and making an appropriate notes about its course.

In all stages of the lesson B. Zel'tserman and N. Rogaleva (Zel'tserman, Rogaleva, 2002) see the main require as visibility and audibility of all pupils of the class. Therefore they consider reasonably carry out the shooting by means of two or three video camera.

Let us express our attitude to the given problem. Certainly this is a good offer. But which school or teacher may permit himself to shoot the lessons during a year. Carrying out definition of research methods - observation, we note that without intervention in the course of teaching process we discount the outer side. A pupil, who sees that he is shot, begins to behave not so as in a real life, inversely he will try to prove himself perfectly. It means that we can only shoot one or in the last resort two-three lessons which is not enough for a rational and full pedagogical diagnostics.

In their articles M. I. Kuznetsova and E. E. Kochurova (Kuznetsova, 1999; Kuznetsova, Kochurova, 2002) expressed their attitude to the set of diagnostic materials determining the preparation of children to education at school. According to their opinion in the course of a pedagogical diagnostics it is important to clarify the following subjects: 1) case of the spatial perception; 2) case of visual perception; 3) case of the motor function and visual-motor coordination; 4) ability of carrying out a classification and extract the features on which it was held; 5) existence intuitive numeral notion; 6) acquisition of notions underlying the count and just with the count (within the notions on operations of summation and subtraction); 7) ability of comparing the two sets on the number of their elements; development of phonemic hearing and notion; 8) generation of precondition towards a successful acquisition of sound analysis and synthesis".

A book named "Pedagogical diagnostics at a practical and experimental affair of a school" (Batrakova, Mosina, Tryapitsyna, 1993) published in Moscow attracted our attention. According to the authors' opinions implementation of a psychological and pedagogical diagnostics requires from a teacher corresponding psychological and diagnostic culture in practice which includes the following components:

1). Psychological literacy takes into account knowledge about general, age and pedagogical psychology, the psychological realities as the objects of diagnostic research, also keep it under focus of attention the impartiality of the teacher during testing and evaluation of his results. Actual requirement of physicians about "causing the threat" for psychologist-teachers that will able to have an influence to the persons passing examinations during testing only explaining the inferences, preventing from development or hurting of psyche.

2). Holding of knowledge on diagnostics, principle of its application by a teacher.

3). Both a teacher and a school psychologist must try to create "a customer situation" and prevent from implementation of a diagnostic research under the "expertise condition" (Batrakova, Mosina, Tryapitsyna, 1993).

\section{Conclusion}

The results of the analyses on pedagogical diagnostics in literature on pedagogy and school practice enable us to come to the conclusion that the pedagogical diagnostics, diagnostics of pupils', teachers', school and form masters' work, in general, diagnostics of the work of all school is imperative. It creates favourable opportunities and conditions for a rational construction of a pedagogical process at an educational centre.

\section{References}

Abbasov, A. N. (2008). Pedagogics: Short conspectus and schemes. Baku: Mutarcim.

Aghayev, A. A. (2006). Pedagogics. Baku: Adiloghlu.

Ahmadov, H. H. (2006). Pedagogics. (An experimental teaching aid). Baku: Azerbaijan International University Press.

Alizada, A. A. (2004). Psychological problems of a modern Azerbaijan school. Baku: Pedagogika (Pedagogics).

Alizada, A. A., \& Alizada, H. A. (2010). Pedagogical psychology. Baku: Azerbaijan State Pedagogical University Press.

Batrakova, I. S., Mosina, A. V., \& Tryapitsyna, A. P. (1993). Pedagogical diagnostics at a practical and experimental affair of a school. A book for teachers. Saint Petersburg: CPS.

Hamzayev, M. A. (1991). Pedagogical psychology. Baku: Maarif.

Ingenkamp, K. (1991). Pedagogical diagnostics (translation from German). Moscow: Pedagogics. 
Kuznetsova, M. I., \& Kochurova, E. E. (2002). Methodology of conducting of pedagogical diagnosis and a set of diagnostical materials determing the children's readiness to the training at a school. Primary school, 3.

Kuznetsova, M. I. (1999). Pedagogical diagnosis of children's readiness to the training at a school and methodical recommendations on the overcoming of the encountered difficulties. Primary school, 8.

Mehrabov, A., Abbasov, A., Zeynalov, Z., \& Hasanov, R. (2006). Pedagogical technologies. Baku: Mutarcim.

Mikhaylichev, E. A. (2003a). Methodology of diagnostics of breeding. School technologies, 4, 123-127.

Mikhaylichev, E. A. (2003b). Methodology of pedagogical psycho-diagnostics. School technologies, 6, $147-157$.

Pashayev, A. Kh., \& Rustamov, F. A. (2007). Pedagogics. A new course. Baku: Nurlan.

Qasimova, L., \& Mahmudova, R. (2002). Pedagogics. Baku: Chashioghlu.

Zel'tserman B., \& Rogaleva, N. (2002). Educational diagnosis of success of training activities. International collection of theoretical, methodological and practical work on education. Education 21st century: achievements and prospects. Ed. V. Zinchenko. Riga: Experiment. 